\title{
Ir(111) Surface State with Giant Rashba Splitting Persists under Graphene in Air
}

\author{
A. Varykhalov, ${ }^{1}$ D. Marchenko, ${ }^{1}$ M. R. Scholz, ${ }^{1}$ E. D. L. Rienks, ${ }^{1}$ T. K. Kim, ${ }^{2, *}$ G. Bihlmayer, ${ }^{3}$ \\ J. Sánchez-Barriga, ${ }^{1}$ and $\mathrm{O}$. Rader ${ }^{1}$ \\ ${ }^{1}$ Helmholtz-Zentrum Berlin für Materialien und Energie, Elektronenspeicherring BESSY II, \\ Albert-Einstein-Straße 15, D-12489 Berlin, Germany \\ ${ }^{2}$ Institute for Solid State Research, IFW Dresden, P.O. Box 270116, D-01171 Dresden, Germany \\ ${ }^{3}$ Peter Grünberg Institut and Institute for Advanced Simulations, Forschungszentrum Jülich and JARA, D-52425 Jülich, Germany
}

(Received 4 August 2011; published 9 February 2012)

\begin{abstract}
We reveal a giant Rashba effect $\left(\alpha_{R} \approx 1.3 \mathrm{eV} \AA\right)$ on a surface state of $\operatorname{Ir}(111)$ by angle-resolved photoemission and by density functional theory. It is demonstrated that the existence of the surface state, its spin polarization, and the size of its Rashba-type spin-orbit splitting remain unaffected when Ir is covered with graphene. The graphene protection is, in turn, sufficient for the spin-split surface state to survive in ambient atmosphere. We discuss this result along with indications for a topological protection of the surface state.
\end{abstract}

DOI: 10.1103/PhysRevLett.108.066804

The Rashba effect is based on symmetry breaking [1]: In the bulk of crystallographically inversion symmetric solids the time-reversal symmetry results in degenerate spin subbands of electronic valence states. At crystal surfaces or interfaces this structural inversion symmetry is broken and the spin degeneracy is lifted. A gradient of the electric potential perpendicular to the surface will then lead to a Rashba effect in two-dimensional systems with large spinorbit coupling [2]. It emerges as a splitting of the band structure into subbands $E_{ \pm}\left(\mathbf{k}_{\|}\right)$of opposite spin which are displaced by electron wave vectors $\pm \Delta \mathbf{k}_{\|}$in opposite directions in momentum space. Regarding the transport properties of the solid this leads to the generation of dissipationless spin currents without the necessity for ferromagnetic materials or magnetic fields $[3,4]$.

In the quantum-mechanical description of a twodimensionally confined electron gas, the Rashba effect is accounted for by the Hamiltonian

$$
H_{R}=\alpha_{R}\left(\sigma_{x} \frac{\partial}{\partial y}-\sigma_{y} \frac{\partial}{\partial x}\right)
$$

where $\sigma$ denotes Pauli spin matrices and the parameter $\alpha_{R}$ is proportional to the potential gradient $\nabla V$ in the $z$ direction and accounts for the size of the spin-orbit interaction. For free electrons, the two spin-split bands $E_{ \pm}\left(\mathbf{k}_{\|}\right)$are described by

$$
E_{ \pm}\left(\mathbf{k}_{\|}\right)=\frac{\hbar^{2} \mathbf{k}_{\|}^{2}}{2 m^{*}} \pm \alpha_{R}\left|\mathbf{k}_{\|}\right|,
$$

where $m^{*}$ is the effective mass. The two $E_{ \pm}\left(\mathbf{k}_{\|}\right)$parabolas are shifted relative to the origin $\bar{\Gamma}\left(\mathbf{k}_{\|}=0\right)$ by a momentum splitting $\Delta k_{\|}=\left(m^{*} \alpha_{R}\right) / \hbar^{2}$.

The Rashba effect has been investigated first and foremost for semiconductor heterostructures [2], reaching Rashba parameters of up to $4 \times 10^{-11} \mathrm{eV} \mathrm{m}$ in InAs-based structures [5]. As for metal surfaces and interfaces,
PACS numbers: 73.22.Pr, 71.70.Ej, 73.20.At, 79.60.Dp

their applications in spintronics are rather conditional and restrained by bulk conductivity of the materials. Nevertheless, metal surfaces remain crucially important model systems for elucidating the physical aspects of the Rashba effect. Spin currents produced by the spin Hall effect in all-metal devices are much larger than in semiconductors [6,7], and very large Rashba effects have been measured for metal surface states which are localized at the outermost atomic layers of the solid-vacuum interface. Discovered by Russian theoretician Tamm in 1932 [8], metal surface states had to wait for their experimental scrutiny until ultrahigh vacuum sample environments became available [9]. They are highly sensitive to adsorbates, and it is hard to conceive an adsorbate that will leave a surface state unaffected while protecting it against the influence of air.

The most prominent example of the Rashba effect at metal surfaces is the $L$-gap surface state on $\mathrm{Au}(111)$ $[10,11]$. Spin- and angle-resolved photoelectron spectroscopy (ARPES) reveal a large spin-orbit splitting $\left(\alpha_{R}=\right.$ $3.3 \times 10^{-11} \mathrm{eV} \mathrm{m}$ ) [10]. Rashba-type splittings were also evidenced in Bi [12] and W(110) covered by monolayers of hydrogen [13], Li [14], Au and Ag [15]. Large spin-orbit effects were recently observed in quantum well states of $\mathrm{Au} / \mathrm{W}(110) \quad\left(\alpha_{R} \approx 1.6 \times 10^{-11} \mathrm{eVm}\right) \quad$ [16] and also $\mathrm{Pb} / \mathrm{Si}(111)\left(\alpha_{R} \approx 4 \times 10^{-12} \mathrm{eV} \mathrm{m}\right)$ [17]. A Rashba effect emerges also in the linear bands of massless Dirac fermions in graphene on a Au monolayer [18]. The term giant Rashba effect has been coined recently for a Rashba parameter $\alpha_{R}$ of the order of $10^{-10} \mathrm{eV}$ m for a surface state of $\mathrm{Bi} / \operatorname{Ag}(111)\left(\alpha_{R} \approx 3.05 \times 10^{-10} \mathrm{eV} \mathrm{m}\right)$ [19]. For most of these states holds that they cannot exist but in ultrahigh vacuum and, therefore, have no apparent practical application. In the present work by means of angle-resolved photoemission we reveal a giant Rashba effect on an $\operatorname{Ir}(111)$ surface state and demonstrate that this surface state 
can persist in ambient atmosphere when the Ir gets protected by a graphene overlayer. Experimental details can be found in the Supplemental Material [20].

Figures 1 and 2 show the characterization of clean and graphene-covered Ir samples. Bare $\operatorname{Ir}(111)$ shows a sharp $p(1 \times 1)$ pattern in low-energy electron diffraction (LEED). The LEED image of graphene/Ir(111) [Fig. 1(b)] reveals a characteristic diffraction pattern with multiple satellite spots which are due to the formation of a superstructure of the moire type which, in turn, is caused by a large misfit $(\sim 10 \%)$ between crystal lattices of graphene and Ir [21]. The high structural quality of the moiré-patterned graphene is also confirmed by direct microscopic characterization by scanning tunneling microscopy [20]. A minor presence of graphene domains rotated by $30^{\circ}$ [22] is also seen [single spots between moiré constellations in Fig. 1(b)].

Figure 1(c) shows the $\operatorname{Ir} 4 f_{5 / 2}$ and $4 f_{7 / 2}$ core levels, both with components originating from inside the bulk and from the surface. The components at $0.5 \mathrm{eV}$ lower binding energy are exclusively due to emission from the topmost atomic layer of $\operatorname{Ir}(111)$ [23], and they were shown by oxygen adsorption to be very sensitive to the local atomic environment [24]. It is, therefore, remarkable that the spectra of the $\operatorname{Ir} 4 f$ core level in Fig. 1(c) cannot be used as an indicator for the presence of graphene on $\operatorname{Ir}(111)$. On the contrary, the formation of graphene on top does not visibly affect the surface component, and line fits show that the energy splitting between bulk and surface components decreases by only $2 \%$ [25]. This indicates very weak graphene-Ir interaction and is in line with the observation that $\operatorname{Ir}(111)$ is a metal substrate that supports the formation of an ideal quasifreestanding electronic structure of graphene [26], similar to the substrate $\mathrm{Au} / \mathrm{Ni}(111)$ [18].

Figures 2(a) and 2(b) compare the overall valence band structure of $\operatorname{Ir}(111)$ before and after formation of graphene, respectively. For graphene/Ir(111) [Fig. 2(b)] one can see
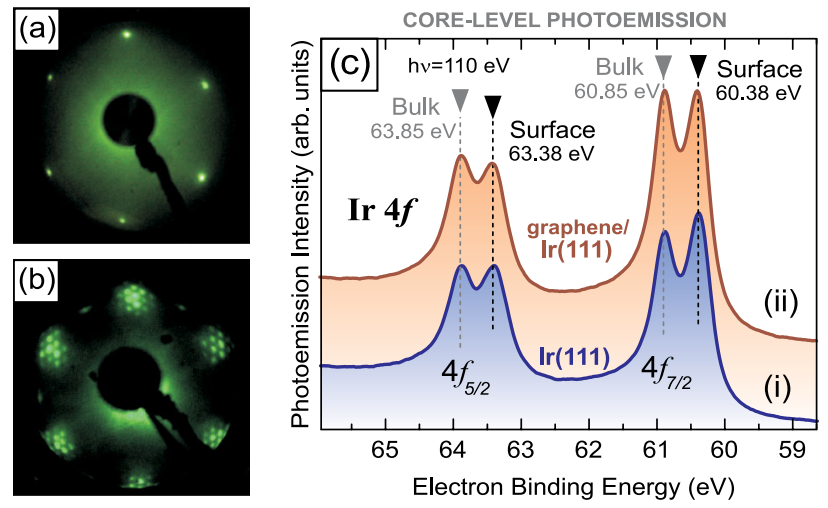

FIG. 1 (color online). Preparation of epitaxial graphene. (a) LEED image of bare $\operatorname{Ir}(111)$ and (b) of moiré-patterned graphene/Ir(111). (b) Comparison of $4 f$ core-level spectra measured from (i) bare $\operatorname{Ir}(111)$ and (ii) graphene/Ir(111). The surface component remains unchanged. strongly dispersing graphene-derived $\pi$ and $\sigma$ states reaching at $\bar{\Gamma}$ binding energies of 8.38 and $3.65 \mathrm{eV}$, respectively. (Note that the $\pi$ and $\sigma$ dispersions are accidentally degenerate with emission from Ir bulk bands.) The high structural quality of the synthesized graphene is additionally confirmed by the observation of a sharp conical dispersion of Dirac fermions at the $\bar{K}$ point [20].

The comparison between Figs. 2(a) and 2(b) delivers another important message: Apparently, the Ir bands preserve their overall dispersions and binding energies upon graphene deposition. This agrees well with the weak interaction between graphene and $\operatorname{Ir}(111)$, noted above. In this
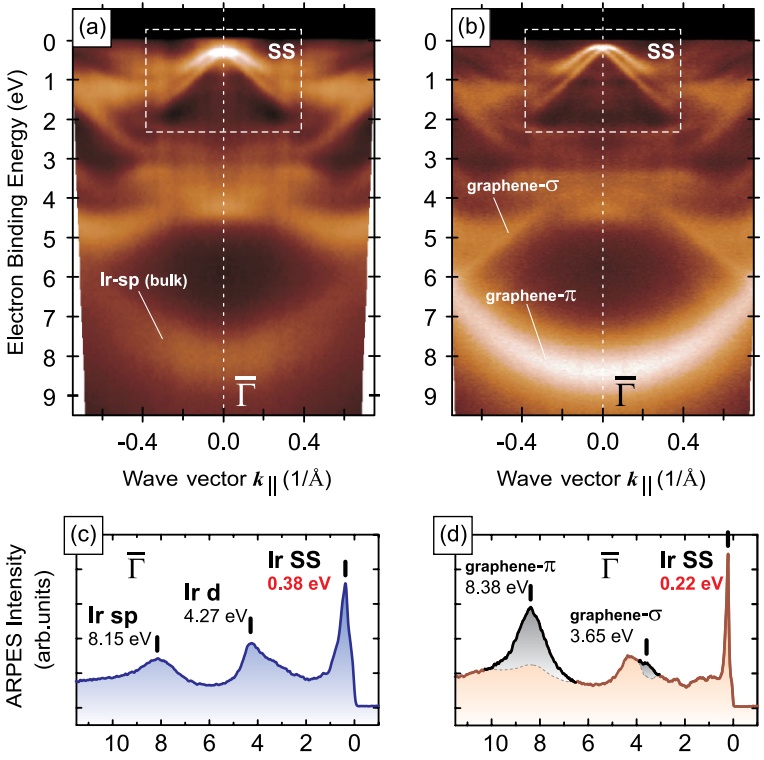

Electron Binding Energy (eV)
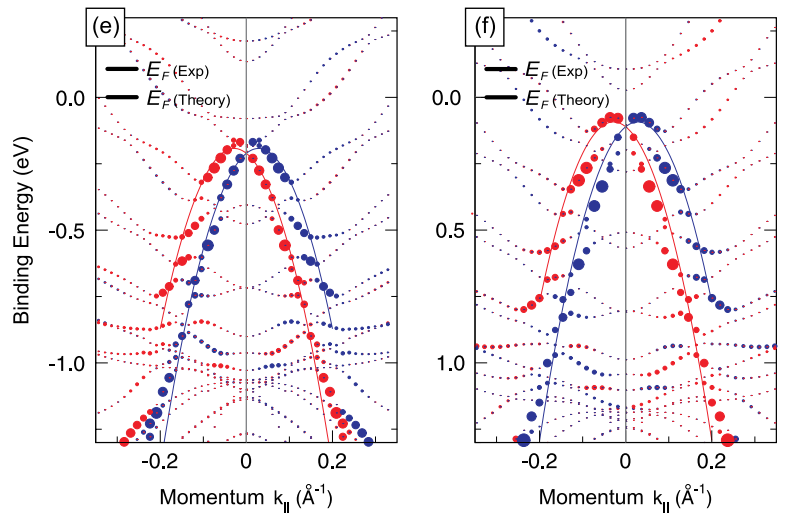

FIG. 2 (color online). Overall valence band structure. (a) Bare $\operatorname{Ir}(111)$ and (b) graphene-covered $\operatorname{Ir}(111)(h \nu=62 \mathrm{eV})$. Spinorbit split surface state (SS) is marked with a white frame. (c), (d) Normal-emission spectra [corresponding to the $\bar{\Gamma}$ point of the surface Brillouin zone] extracted from (a) and (b), respectively. The spectra allow for a quantitative comparison between intensities of Ir surface state and graphene bands. (e) Band structure calculation for $\operatorname{Ir}(111)$ showing the spin-orbit split surface state without and (f) with graphene on top with identical Rashba splittings. 
context, very intriguing is the band showing up at $0.2-$ $0.3 \mathrm{eV}$ below $E_{F}$ at $\bar{\Gamma}$ [the region is marked by a frame and the dispersion is denoted as SS in Figs. 2(a) and 2(b)]. From studies at $\bar{\Gamma}$ this band is known to be an $\operatorname{Ir}(111)$ surface state situated at $0.4 \mathrm{eV}$ binding energy above the bulk $L_{6-}$ point at $1 \mathrm{eV}$ binding energy [27]. The state is related to the $L$-gap surface states of $\mathrm{Au}(111)$ but in Ir the bulk bands have a different order so that the surface state is reversed (i.e., with effective mass $m^{*}<0$ ). It is seen now that this surface state is also clearly split in $\mathbf{k}_{\|}$, resembling classical Rashba-type spin-orbit split surface states [10]. The splitting amounts to $2 \Delta k_{\|}=0.075 \AA^{-1}$. The population of this state remains unaffected by the formation of graphene on $\operatorname{Ir}(111)$. This can be seen quantitatively in normal-emission $(\bar{\Gamma})$ spectra plotted in Figs. 2(c) and 2(d). We paid attention explicitly to the splitting sampling the surface-state region with enhanced angular and energy resolution. The dispersion acquired for bare $\operatorname{Ir}(111)$ is shown in Fig. 3(a). Indeed, the electronic state is composed of two identical parabolic bands with a Rashba parameter of $\alpha_{R} \approx 1.3 \times 10^{-10} \mathrm{eVm}$. The binding energy of the top of the bands is determined as $340 \mathrm{meV}$. Figure 3(b) demonstrates what happens to the split state when graphene is grown on top: The only change concerns the binding energy of the parabolic bands, which decreases by $150 \mathrm{meV}$ to $190 \mathrm{meV}$. (The different sharpness of the bands is not a systematic effect.) This is clear evidence that the split band is a surface state of $\operatorname{Ir}(111)$ which persists under graphene, but is not affected by the graphene band structure. Such behavior is quite different from that of $d$-type surface states of $\operatorname{Ir}$ emerging at the $\bar{K}$ point of $\operatorname{Ir}(111)$ which apparently interact with the $\pi$ band of graphene [22]. It is also interesting to note that neither binding energy nor the spin splitting of the Ir surface state under graphene depends on rotational variants [22] of graphene on $\operatorname{Ir}(111)$.

Density functional calculations [20] with spin-orbit interaction performed for a 15-atomic-layer-thick slab of Ir with graphene placed at a distance of $3.5 \AA$ and expanded to the $\operatorname{Ir}(111)$ lattice constant confirm the presence of a $p_{z}$-type surface state; see Fig. 2(e). A large symbol size indicates surface localization and gray (red and blue) indicates the spin. Apart from a small shift of $0.1 \mathrm{eV}$ towards
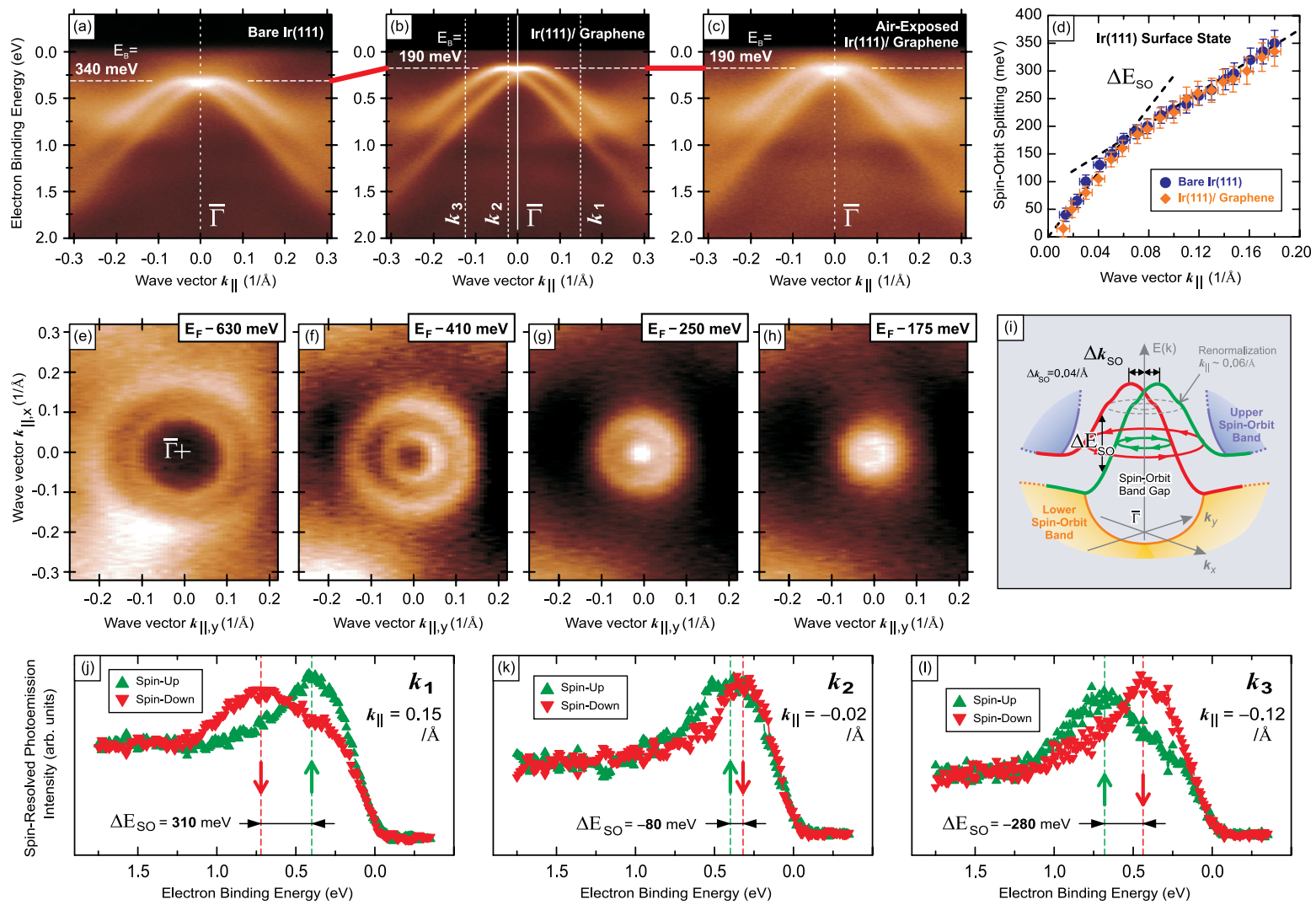

FIG. 3 (color online). Characterization of the spin-orbit split surface state on $\operatorname{Ir}(111)$. Dispersion of surface state on (a) bare $\operatorname{Ir}(111)$ and (b) under graphene grown on $\operatorname{Ir}(111)$. (c) Dispersion of the surface state under graphene on $\operatorname{Ir}(111)$ after exposure to ambient atmosphere for $15 \mathrm{~min}$. (d) Detailed comparison of spin-orbit splitting $\Delta E_{\mathrm{SO}}\left(k_{\|}\right)$for bare and graphene-covered $\operatorname{Ir}(111)$. (e)(h) Constant energy surfaces of the Ir surface state extracted from full photoemission mapping around $\bar{\Gamma}$. In agreement with the Rashba model, two circles are revealed corresponding to opposite directions of spin circulation. (i) Sketch of spin-orbit splitting of the surface state indicating topological protection. (j)-(l) Direct proof of spin splitting of the Ir surface state under graphene by spin-resolved photoemission. Measured spectra correspond to wave vectors $k_{1}, k_{2}$, and $k_{3}$ as denoted in (b). The photon energy was 62 eV. 
the Fermi level, the surface state is hardly influenced by the graphene adsorption in the calculation [Fig. 2(f)].

Results of a full photoemission mapping of the Ir surface state under graphene are presented in Figs. 3(e)-3(h) as a sequence of constant energy surfaces cut at binding energies of $630,410,250$, and $175 \mathrm{meV}$. The maps are fully consistent with the Rashba model of spin-orbit splitting: For higher binding energies two circles are observed which correspond to opposite directions of spin circulation [arrowheads in Fig. 3(i)]. At $\sim 250 \mathrm{meV}$, the inner circle shrinks to a point which is the Kramers point of spin degeneracy, and for $175 \mathrm{meV}$ binding energy only one circle is left, which corresponds to the only direction of spin rotation. This mapping also shows that two bands that disperse in parallel from the zone center as have been obtained from $\operatorname{Ir}(111)$ recently [28] can easily result from a slight misalignment of the experimental setup.

We have additionally confirmed this Rashba scenario through direct observation of the spin. Spin-resolved spectra measured for the emission angles corresponding to the electron wave vectors $k_{1}, k_{2}$, and $k_{3}$ [labels of Fig. 3(b)] are displayed in Figs. 3(j)-3(1). Indeed, the splitting of the bands is a spin splitting. The splitting $\Delta E_{\mathrm{SO}}$ is large for $k_{1}>0$ far from $\bar{\Gamma}$, decreases for $k_{2}$ closer to $\bar{\Gamma}$, and, expectedly, reverses for $k_{3}<0$.

We have fitted the surface-state dispersions shown in Figs. 3(a) and 3(b) and investigated how the spin-orbit splitting $\Delta E_{\mathrm{SO}}$ behaves with wave vector $k_{\|}$for bare $\operatorname{Ir}(111)$ and for graphene-covered $\operatorname{Ir}(111)$. Figure 3(d) shows that in both cases $\Delta E_{\mathrm{SO}}\left(k_{\|}\right)$is linear, corresponding to parabolic, free-electron-like bands, and fulfills the criterion $\Delta E_{\mathrm{SO}}\left(k_{\|}=0\right)=0$ of the Rashba effect. However, $\Delta E_{\mathrm{SO}}$ suffers a kink at $k_{\|}=0.06 \AA^{-1}$. This is ascribed to the interaction of the surface state with Ir bulk $d$ bands in this range because the surface states are partially degenerate with bulk bands [27] and become three dimensional.

The other surprising information available from Fig. 3(d) is a quantitative equality of the Rashba splitting $\Delta E_{\mathrm{SO}}$ for bare $\operatorname{Ir}(111)$ and for graphene/Ir(111). According to a recent analysis [29] there is a large and universal potential step $\Delta V$ at any graphene-metal interface which can be estimated for graphene/ $\operatorname{Ir}(111)$ from the work function of $\operatorname{Ir}(111)(5.8 \mathrm{eV})$, work function of graphene $(4.5 \mathrm{eV})$, and from the Fermi-energy shift $\Delta E_{F}$ $(0.1 \mathrm{eV})$ as $1.2 \mathrm{eV}$. The surface or interface potential gradient has been assigned an important role for the Rashba effect $[11,13,14]$. Based on Li/W(110) adsorption experiments, the spin-orbit splitting has even been suggested as a local probe of surface potential gradients [14]. Previously, we found no such influence for $\mathrm{Au} / \mathrm{W}(110)$ and related systems [16]. Here, the change in surface potential modifies the binding energy but not the spin-orbit splitting of the present surface state. This is also confirmed by our $a b$ initio calculations (Fig. 2): $\alpha_{R}$ is $1.30 \mathrm{eV} \AA$ with and without graphene despite the change in the work function by $0.84 \mathrm{eV}$. The splitting of the state is therefore ascribed to the large nuclear number of the ${ }_{77} \mathrm{Ir}$. It is insensitive to the change in the surface potential due to the graphene adsorption as long as the asymmetry of the wave function is not modified [10(d),11]. This insensitivity can be verified from the charge-density plots in Fig. S5 [20]. It can further be seen that the Ir surface state possesses $p_{z}$-like orbital character close to the $\bar{\Gamma}$ point of $\operatorname{Ir}(111)$ only. Away from $\bar{\Gamma}$ it has $d_{x, z}$-like character and behaves as a deeply bulk penetrating surface resonance [20].

Before closing, we want to address the reason for the robustness of the surface state. Recently, topological surface states have been predicted and observed which are spin-orbit split and protected by time-reversal symmetry [30-32], and the question is whether the presently encountered stability of the surface state towards graphene deposition is related. It was pointed out recently [31] that the spin-polarized and Rashba-split $L$-gap surface state of $\mathrm{Au}(111)$ does not fulfil the criterion for topological protection, which is an even number of Fermi level crossings between two time-reversal invariant $\mathbf{k}$ points of the surface Brillouin zone, as occurs with the surface state identified on $\mathrm{Bi}_{2} \mathrm{Se}_{3}$ [31]. Unlike on $\mathrm{Au}$, the present Ir surface state becomes degenerate with bulk $d$ bands, and its tails connect to bulk $d$ bands as seen in Fig. 2. Acquiring the dispersion of Ir bands with $\mathbf{k}_{\perp}$ by performing photoemission measurements with variable photon energy, we demonstrate that this state connects bulk states of Ir across a bulk band gap [20].

While it apparently does not fulfil the criterion for topological insulators of an even number of crossings of $E_{F}$ between the two time-reversal invariant $\mathbf{k}$ points $\bar{\Gamma}$ and $\bar{M}$, the criterion for topological protection, if adopted to a metal, has to consider instead of the Fermi level a curved line lying between the relevant bulk bands, and such a line is crossed by the surface state in fact only an odd number of times between $\bar{\Gamma}$ and $\bar{M}$ [20]. A similar situation has been found for the (111) surface of Sb recently [32], but the Ir is a more complicated case for which the topological properties such as the $Z_{2}$ invariant cannot be obtained theoretically at present.

Finally, considering the ability of the spin-split surface state to exist under graphene, we have tested how well graphene may protect the Ir surface state from the environment. We have exposed a graphene-covered $\operatorname{Ir}(111)$ sample to ambient atmosphere for $15 \mathrm{~min}$ and measured the dispersion of the surface state right after this. The result is presented in Fig. 3(c). Although a somewhat stronger background is seen due to remaining adsorbates, an accurate analysis shows that neither binding energy nor spin splitting of the surface state is influenced by the air [Fig. 3(d)]. In a technological context this means that graphene, weakly interacting with its substrate, can be considered an ideal capping layer, which on the one hand 
protects metallic surfaces from a chemically reactive environment $[20,33]$, and on the other hand keeps its electronic and spin structure intact even at the atomic level of surface electronic states which are most promising generators of two-dimensional spin currents.

In summary, we have demonstrated in experiment and theory (i) a previously unknown Rashba-type splitting of giant size of a surface state on $\operatorname{Ir}(111)$, (ii) that the surface state survives when $\operatorname{Ir}(111)$ becomes covered with epitaxial graphene while it changes its binding energy by $150 \mathrm{meV}$, (iii) that neither dispersion nor Rashba splitting of the surface state is influenced by the presence of graphene on top of $\operatorname{Ir}(111)$, (iv) that the spin-dependent band dispersion is consistent with topological protection, and we showed that (v) graphene protects the surface electronic structure of $\operatorname{Ir}(111)$ so well that the spin-orbit split surface state survives the exposure of the sample to ambient atmosphere.

This work was supported by SPP 1459 of the Deutsche Forschungsgemeinschaft.

*Present address: Diamond Light Source, Didcot, Oxfordshire, OX11 0DE, United Kingdom.

[1] E. I. Rashba, Fiz. Tverd. Tela (Leningrad) 2, 1224 (1960); [Sov. Phys. Solid State 2, 1109 (1960)]; Y. A. Bychkov and E. I. Rashba, J. Phys. C 17, 6039 (1984).

[2] R. Winkler, Spin-Orbit Coupling Effects in TwoDimensional Electron and Hole Systems (Springer, Berlin, 2003).

[3] S. Murakami, N. Nagaosa, and S.-C. Zhang, Science 301, 1348 (2003).

[4] J. Sinova, D. Culcer, Q. Niu, N. A. Sinitsyn, T. Jungwirth, and A. H. MacDonald, Phys. Rev. Lett. 92, 126603 (2004).

[5] M. W. Wu, J. H. Jiang, and M. Q. Weng, Phys. Rep. 493, 61 (2010).

[6] T. Kimura, Y. Otani, T. Sato, S. Takahashi, and S. Maekawa, Phys. Rev. Lett. 98, 156601 (2007).

[7] T. Seki, Y. Hasegawa, S. Mitani, S. Takahashi, H. Imamura, S. Maekawa, J. Nitta, and K. Takanashi, Nature Mater. 7, 125 (2008).

[8] I. Tamm, Phys. Z. Sowjetunion 1, 733 (1932).

[9] E. W. Plummer and W. Eberhardt, Adv. Chem. Phys. 49, 533 (1982).

[10] (a) S. LaShell, B. A. McDougall, and E. Jensen, Phys. Rev. Lett. 77, 3419 (1996); (b) G. Nicolay, F. Reinert, S. Hüfner, and P. Blaha, Phys. Rev. B 65, 033407 (2001); (c) M. Hoesch, M. Muntwiler, V. N. Petrov, M. Hengsberger, L. Patthey, M. Shi, M. Falub, T. Greber, and J. Osterwalder, Phys. Rev. B 69, 241401(R) (2004); (d) G. Bihlmayer, Yu. M. Koroteev, P. M. Echenique, E. V. Chulkov, and S. Blügel, Surf. Sci. 600, 3888 (2006).

[11] L. Petersen and P. Hedegård, Surf. Sci. 459, 49 (2000).

[12] T. Hirahara, K. Miyamoto, I. Matsuda, T. Kadono, A. Kimura, T. Nagao, G. Bihlmayer, E. V. Chulkov, S. Qiao, K. Shimada, H. Namatame, M. Taniguchi, and S. Hasegawa, Phys. Rev. B 76, 153305 (2007).
[13] M. Hochstrasser, J.G. Tobin, E. Rotenberg, and S.D. Kevan, Phys. Rev. Lett. 89, 216802 (2002).

[14] E. Rotenberg, J. W. Chung, and S. D. Kevan, Phys. Rev. Lett. 82, 4066 (1999).

[15] A. M. Shikin, A. Varykhalov, G. V. Prudnikova, D. Usachev, V. K. Adamchuk, Y. Yamada, J. Riley, and O. Rader, Phys. Rev. Lett. 100, 057601 (2008).

[16] A. Varykhalov, J. Sánchez-Barriga, A. M. Shikin, W. Gudat, W. Eberhardt, and O. Rader, Phys. Rev. Lett. 101, 256601 (2008).

[17] J.H. Dil, F. Meier, J. Lobo-Checa, L. Patthey, G. Bihlmayer, and J. Osterwalder, Phys. Rev. Lett. 101, 266802 (2008).

[18] A. Varykhalov, J. Sánchez-Barriga, A. M. Shikin, C. Biswas, E. Vescovo, A. Rybkin, D. Marchenko, and O. Rader, Phys. Rev. Lett. 101, 157601 (2008).

[19] C. R. Ast, J. Henk, A. Ernst, L. Moreschini, M. C. Falub, D. Pacilé, P. Bruno, K. Kern, and M. Grioni, Phys. Rev. Lett. 98, 186807 (2007).

[20] See Supplemental Material at http://link.aps.org/ supplemental/10.1103/PhysRevLett.108.066804 for more details.

[21] A. T. N'Diaye, J. Coraux, T. N. Plasa, C. Busse, and T. Michely, New J. Phys. 10, 043033 (2008).

[22] E. Starodub, A. Bostwick, L. Moreschini, S. Nie, F. E. Gabaly, K. F. McCarty, and E. Rotenberg, Phys. Rev. B 83, 125428 (2011).

[23] J. F. van der Veen, F. J. Himpsel, and D. E. Eastman, Phys. Rev. Lett. 44, 189 (1980).

[24] M. Bianchi, D. Cassese, A. Cavallin, R. Comin, F. Orlando, L. Postregna, E. Golfetto, S. Lizzit, and A. Baraldi, New J. Phys. 11, 063002 (2009).

[25] P. Lacovig, M. Pozzo, D. Alfé, P. Vilmercati, A. Baraldi, and S. Lizzit, Phys. Rev. Lett. 103, 166101 (2009).

[26] I. Pletikosić, M. Kralj, P. Pervan, R. Brako, J. Coraux, A. T. N'Diaye, C. Busse, and T. Michely, Phys. Rev. Lett. 102, 056808 (2009).

[27] J. F. van der Veen, F. J. Himpsel, and D. E. Eastman, Phys. Rev. B 22, 4226 (1980).

[28] I. Pletikosić, M. Kralj, D. Šokčević, R. Brako, P. Lazić, and P. Pervan, J. Phys. Condens. Matter 22, 135006 (2010).

[29] G. Giovannetti, P.A. Khomyakov, G. Brocks, V. M. Karpan, J. van den Brink, and P. J. Kelly, Phys. Rev. Lett. 101, 026803 (2008).

[30] C. L. Kane and E. J. Mele, Phys. Rev. Lett. 95, 226801 (2005); B. A. Bernevig and S. C. Zhang, Phys. Rev. Lett. 96, 106802 (2006); M. König et al., Science 318, 766 (2007); J.E. Moore and L. Balents, Phys. Rev. B 75, 121306 (2007); R. Roy, Phys. Rev. B 79, 195322 (2009); S. Murakami, New J. Phys. 9, 356 (2007).

[31] Y. Xia, D. Qian, D. Hsieh, L. Wray, A. Pal, H. Lin, A. Bansil, D. Grauer, Y. S. Hor, R. J. Cava, and M. Z. Hasan, Nature Phys. 5, 398 (2009).

[32] D. Hsieh et al., Science 323, 919 (2009).

[33] V. A. Mozhayskiy, A. Y. Varykhalov, A. G. Starodubov, A. M. Shikin, S. I. Fedoseenko, and V. K. Adamchuk, Phys. Low-Dim. Struct. (2003) 105. 